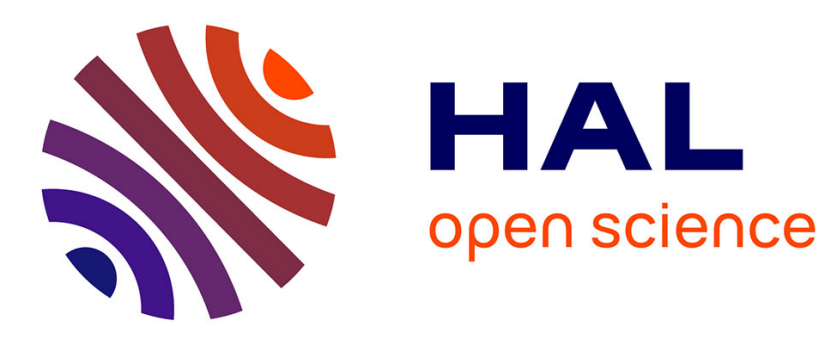

\title{
Micromechanical analysis of second order work in granular media
}

Nejib Hadda, François Nicot, Franck Bourrier, Luc Sibille, Farhang Radjai, Félix Darve

\section{- To cite this version:}

Nejib Hadda, François Nicot, Franck Bourrier, Luc Sibille, Farhang Radjai, et al.. Micromechanical analysis of second order work in granular media. Granular Matter, 2013, 15 (2), pp.221-235. 10.1007/s10035-013-0402-3 . hal-01916016

\section{HAL Id: hal-01916016 https://hal.science/hal-01916016}

Submitted on 8 Nov 2018

HAL is a multi-disciplinary open access archive for the deposit and dissemination of scientific research documents, whether they are published or not. The documents may come from teaching and research institutions in France or abroad, or from public or private research centers.
L'archive ouverte pluridisciplinaire HAL, est destinée au dépôt et à la diffusion de documents scientifiques de niveau recherche, publiés ou non, émanant des établissements d'enseignement et de recherche français ou étrangers, des laboratoires publics ou privés. 


\title{
Micromechanical analysis of second order work in granular media
}

\author{
Nejib Hadda • François Nicot • Franck Bourrier • \\ Luc Sibille · Farhang Radjai · Félix Darve
}

\begin{abstract}
This paper examines instabilities in granular materials from a microscopic point of view through numerical simulations conducted using a discrete element method on two three-dimensional specimens. The detection and the tracking of grain scale deformation mechanisms constitute the key point for a better understanding the failure process and puzzling out what lies behind the vanishing of the macroscopic second order work. For this purpose, the second order work from microscopic variables, involving contact force and branch vector, was introduced and tracked numerically. Then, all contacts depicting negative values of the second order work were deeply investigated, especially their spatial distribution (homogeneity, agglomeration, dispersion...) within the specimen according to the density of the granular assembly and to the loading direction. A set of comparisons has been considered in this context in order to highlight how a specimen is populated with such contacts whether it is loaded along a direction included within the plastic tensorial zone or along a direction for which the specimen is likely to behave elastically (elastic tensorial zone). Moreover, these comparisons concerned also loading directions within the cone of instability so that links between the vanishing
\end{abstract}

N. Hadda $(\varangle) \cdot$ F. Nicot $~$ F. Bourrier

Irstea, Grenoble, France

e-mail: nejib.hadda@irstea.fr

L. Sibille

Institut de Recherche en Génie Civil et Mécanique,

CNRS, LUNAM Université, Nantes, France

F. Radjai

Laboratoire de Mécanique et de Génie Civil,

Université de Montpellier, Montpellier, France

F. Darve

Laboratoire Sols Solides Structures Risques,

UJF-INPG-CNRS, Grenoble, France of both microscopic and macroscopic second order works can be established and the local mechanisms responsible for failure occurrence may be figured out.

Keywords Microscopic variables · Grain scale $\cdot$ Second order work - Granular material - Discrete element method . Homogeneity · Spatial distribution · Instability

\section{Introduction}

Concern about failure in geomaterials and eligible interparticle mechanisms occurring during its process is still generating considerable research interest and raising many issues, therefore it is desirable to understand this phenomenon as well as possible.

Micromechanics is likely to lie in the heart of many aspects of failure in granular media $[11,20,33,36]$ nevertheless it is worth noting that, for granular materials, the macro and micro worlds are widely connected. On one hand, when applying an external force on the boundaries of an assembly of grains (macro scale), this force is transmitted via the contact network from particles in contact with the boundaries to the core of the specimen generating changes in inter-particles contact forces which in turn guide the local motions of particles (translational and rotational displacements) through constitutive equations and physical constraints (contact law, internal angle of friction, degrees of freedom, non uniform distribution of local variables...) giving rise to the macroscopic deformation. On the other hand, failure is likely to be set off at the micro scale through local instabilities inside the assembly before it amplifies to concern the whole specimen and can be detected thereafter at the macro scale by an outburst in kinetic energy which may be followed by the vanishing of the stresses on the boundaries and a brutal collapse of the 
specimen (large deformations) if the loading is stress or force controlled.

In recent years, increasing researches and approaches have been focused at the micro scale with a fundamental aim to physically describe the micromechanical behavior and/or to intercept inter-particles' or structure's like scenarios governing the failure process $[28,37]$. The description of the micromechanical behavior covers the description of the structure (positions of grains and contact points), as well as the local kinematics and its evolutions [6,33] (displacements, rotations. ..) and also the description of inter-particles' contact forces network including force chains [28,29].

The local descriptions mentioned above depend on each other and were investigated by many authors numerically and experimentally for different purposes either to validate micro-macro mechanical approaches and constitutive models $[2,11,14,17,24,26,28]$ or to predict the occurrence of shear bands $[1,5,12,13]$ or instabilities [35,38]. In this regard, Radjai et al. [9], Oda et al. [24], Oda and Iwashita [25] and Iwashita and Oda [28] focused on contact networks, strong and weak force networks (including or not force chains networks), Kuhn and Bagi [10,12,13] were interested in defining and describing objective and non-objective relative motions (particularly rotations at different scales) and distinguishing their contributions in the deformation of a granular assembly, Tordesillas [33] and Tordesillas and Muthuswamy [34] showed that the presence of 3-cycles in a contact network is associated with frustrated rotations of particles, and confirmed the influence of particles sliding and relative rotations on the stability and buckling of force chains. However, a quantitative micro-macro description is still being sought, that's why it is crucial to put forward an efficient grain-scale tool in order to investigate physical origins of failure and ideally if it can be related to an instability criterion such as the vanishing of the so called second order work.

Indeed the vanishing of the second order work [8] constitutes a basic and necessary condition for failure to occur $[18,19,21]$ but also gives indications about the specific stress or strain loading directions that may lead a system to failure [19]. On this basis, a link between the second order work from macroscopic variables and the second order work from microscopic variables involving contact forces and particles' positions was developed [20] in order to address failure from the micromechanical point of view and describe the local mechanisms governing the vanishing of the second order work.

In this paper, the microscopic formalism of the second order work in granular material is reviewed with a brief sketch over some theoretical aspects lying behind its establishment. Then numerical simulations using the discrete element method on two three dimensional specimens are performed in order to validate the theoretical development. Agreements between theoretical and numerical results and between macro and micro second order works are both discussed in the second part.

Finally some microscopic investigations related to the spatial distribution of contacts with negative values of microscopic second order work are carried out in the last session. This distribution is related to the diffuse nature of the failure considered here, highlighting some micro-structural aspects (or origins) of this latter. This last analysis also offers the opportunity to intercept major grain-scale clues lying behind the vanishing of the macroscopic second order work.

\section{Extension of the second order work to the micro-structure}

The second order work [8] can be either expressed through the material description (Lagrangian formalism) as $W_{2}=$ $\int_{V_{o}} \delta \Pi_{i j} \delta F_{i j} d V_{o}$ or through the spatial description (Eulerian formalism) by assuming small deformations and neglecting geometrical aspects $[18,22]$ as $W_{2}=\int_{V} \delta \sigma_{i j} \delta \varepsilon_{i j} d V$, where $\overline{\bar{\Pi}}$ is the first Piola-Kirchoff stress tensor, $\overline{\bar{F}}$ the general term of the deformation gradient tensor, $\overline{\bar{\sigma}}$ the Cauchy stress tensor and, and $\overline{\bar{\varepsilon}}$ is the strain tensor. In both descriptions, the second order work is associated with incremental evolutions involving incremental pair quantities $\delta F_{i j}$ and $\delta \Pi_{i j}$ or $\delta \varepsilon_{i j}$ and $\delta \sigma_{i j}$ related through the mechanical constitutive behavior of the granular packing. $V$ is the current volume of the system and $V_{0}$ refers to the volume at the initial configuration.

It was shown [20] that the second order work can be expressed in terms of the microscopic variables accounting for the microstructure of the material.

Considering a granular assembly composed of $N$ particles ' $p$ ' $(1 \leq p \leq N)$, the second order work from microscopic variables writes

$W_{2}^{\mu}=\sum_{p, q} \delta f_{i}^{c} \delta l_{i}^{c}+\sum_{p \in V} \delta f_{i}^{p} \delta x_{i}^{p}$

where $\bar{l}^{c}$ is the branch vector relating the centers of contacting particles $p$ and $q, \bar{f}^{c}$ the inter-particle contact force, $\bar{x}^{p}$ the position of particle ' $p$ ' and $\bar{f}^{p}$ the resultant force applied to the particle ' $p$ '.

Equation (1) shows that the micromechanical expression of the second-order work is the combination of two terms. The first term $W_{2}^{m}=\sum_{p, q} \delta f_{i}^{c} \delta l_{i}^{c}$, can be related to the local second-order work $\sum_{p, q} \delta f_{i}^{c} \delta u_{i}^{c}$ (where $\delta u^{c}$ is the inter-particle relative displacement, Nicot and Darve [23]), and connect the contact force network with the geometrical distribution of branches between grains.

The second term $\sum_{p \in V} \delta f_{i}^{p} \delta x_{i}^{p}$ of Eq. (1) involves the incremental unbalanced force $\delta \bar{f}^{p}$ applied to each particle ' $p$ ' and the incremental change in position of particle ' $p$ '.

The numerical simulations performed herein are kinematically controlled, they are thus not accompanied by a burst 
in kinetic energy and inertial effects can hardly occur. The whole analysis can be done in quasi static regime and the contribution of the term $\sum_{p \in V} \delta f_{i}^{p} \delta x_{i}^{p}$ becomes negligible. Equation (1) therefore reduces to

$W_{2}^{\mu}=\sum_{p, q} \delta f_{i}^{c} \delta l_{i}^{c}$

When important particles rearrangements occur in irreversible strain regime (sliding between particles, contact opening and contact creation), the second term is likely to be no longer negligible and its contribution may be considerable.

In the next part, we will focus on the comparison between macro and micro second order works and on the numerical validation of Eq. (2) analytically developed above.

\section{Numerical analysis using discrete element method}

\subsection{Discrete element model}

In what follows, the incremental variation of a given variable $\xi$ denoted by $\delta \xi(\delta \xi(t)=\dot{\xi}(t) \delta t)$ during an infinitesimal variation of time $t$, is approximated by finite increments in the discrete elements method, thus will be rather denoted by $\Delta \xi$.

The vanishing of the second order work was proved to be related to both porosity and confining pressure of the assembly $[21,30,31]$. In order to cover as many cases as possible and generalize the results found in this paper independently from such parameters, numerical simulations were conducted on two three-dimensional specimens (Fig. 1a) of densely (porosity $=0.38$ ) and loosely (porosity $=0.42$ ) compacted assembly of particles $S_{1}$ and $S_{2}$ respectively. We used the software 'Yade' [40] based on the discrete element method [3].

The motion of the particles is governed by the elongation of both a normal and a tangential spring created at the initial contact point of each pairwise overlapping particles (see Fig. 1b).

In the normal direction, the contact constitutive relation is linear elastic and it assumes that the normal component $f_{n}^{c}$ of the contact force is linearly related to the particle overlap through the normal contact stiffness denoted by $k_{n}$.

The tangential component $f_{t}^{c}$ of the contact force involves similarly the tangential contact stiffness denoted by $k_{t}$ and the tangential relative displacement which can be calculated by integrating the relative tangential velocity $v_{t}$ in the contact plane during the lifetime of the overlap.

The friction is incorporated at the contact level by the Coulomb friction law, thus $f_{t}^{c}$ must obey the constraint $f_{t}^{c} \leq \mu f_{n}^{c}$, where $\mu$ is the coefficient of friction. When this limit is reached, the tangential relative motion is regarded as sliding with a friction force $\mu f_{n}^{c}$ directed opposite to the tangential relative velocity. Thus, in the tangential direction, the constitutive relation is linear elastic-purely plastic.

Moreover, for cohesionless materials, the springs act until the bodies depart from each others and come out of contact, only compressive normal forces are allowed.

\subsection{Numerical simulations}

The two specimens are cubical in shape and contain 10,000 spherical particles of uniform radius distribution ranging from 2 to $12 \mathrm{~mm}$ enclosed within six rigid frictionless walls.

The parameters in the contact constitutive relation are chosen such that $k_{n} / D=356(\mathrm{MPa})$ and $k_{t} / k_{n}=0.42$, where $D$ is the mean diameter of the two particles in contact. Interparticle friction angle $\varphi_{c}$ is set to $35^{\circ}$. The characteristics and mechanical parameters of both specimens are detailed in Table 1.

Both assemblies were compacted from initially sparse arrangements of particles to an isotopic state by increasing particles sizes until the desired isotropic pressure $\left(\sigma_{1}=\sigma_{3}=\right.$
Fig. 1 A three dimensional view of the specimen (a), the inter-particle contact law (b)

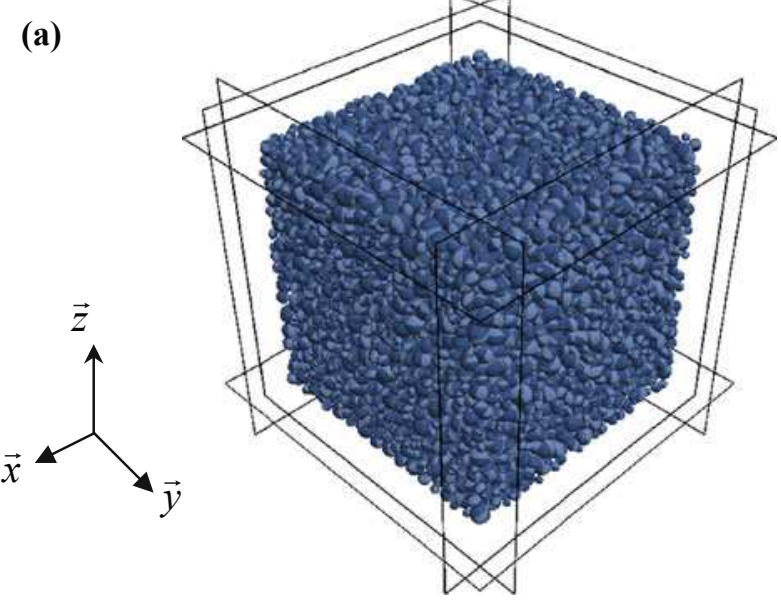

(b)

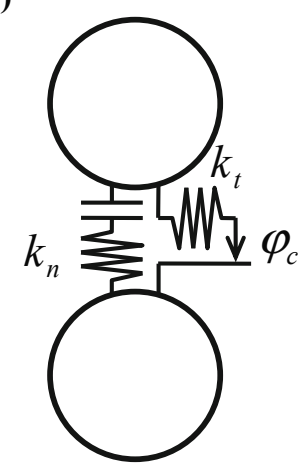


Table 1 Physical and mechanical parameters of the dense and loose specimens $S_{1}$ and $S_{2}$

\begin{tabular}{llllllll}
\hline & $k_{n} / D[\mathrm{MPa}]$ & $k_{t} / k_{n}$ & $\varphi_{c}\left[^{\circ}\right]$ & $\begin{array}{l}\text { Particle density }(\rho) \\
{\left[\mathrm{kg} / \mathrm{m}^{3}\right]}\end{array}$ & $\begin{array}{l}\text { Void ratio } \\
(e)\end{array}$ & $\begin{array}{l}\text { Coordination number } \\
(z)\end{array}$ & $\begin{array}{l}\text { Reduced stiffness } \kappa \\
\left(\kappa=k_{n} /(D p)\right)\end{array}$ \\
\hline $\mathrm{S}_{1}$ & 356 & 0.42 & 35 & 3,000 & 0.636 & 4.43 & $1,780(p=200 \mathrm{kPa})$ \\
$\mathrm{S}_{2}$ & 356 & 0.42 & 35 & 3,000 & 0.71 & 4.22 & $3,560(p=100 \mathrm{kPa})$ \\
\hline
\end{tabular}
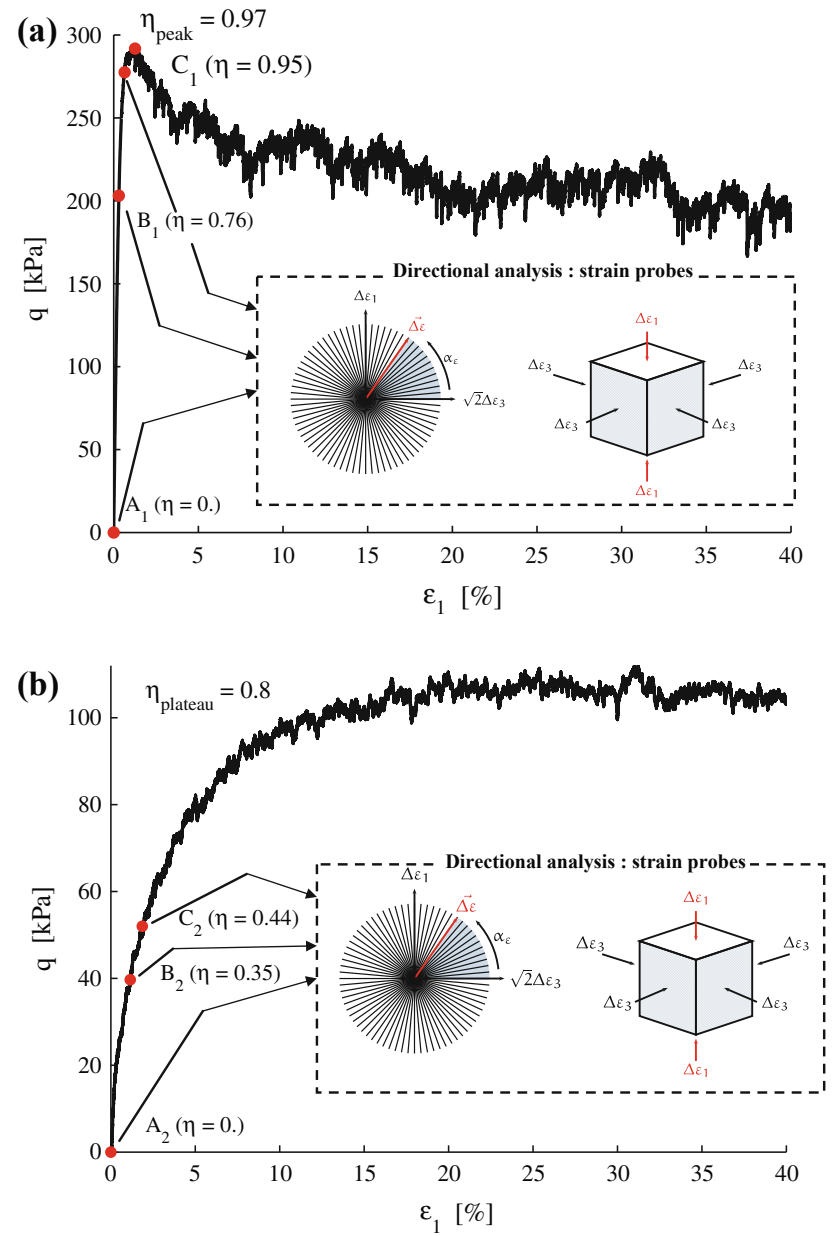

Fig. 2 Deviatoric stress in terms of axial strain at a confining pressure of $200 \mathrm{kPa}$ for $\mathrm{S} 1(\mathbf{a})$, and $100 \mathrm{kPa}$ for S2 (b)

$200 \mathrm{kPa}$ for $S_{1}$ and $\sigma_{1}=\sigma_{3}=100 \mathrm{kPa}$ for $S_{2}$ ) is reached. They are then subjected to an axisymmetric drained triaxial compression.

The evolution of both deviatoric stress $q=\sigma_{1}-\sigma_{3}$ and volumetric strain $\varepsilon_{v}$ versus the axial strain $\varepsilon_{1}$ are shown in Figs. 2 and 3 respectively for both specimens.

For the loose specimen, the deviatoric stress increases continuously (positive hardening regime) toward a limit plateau at which $q=110 \mathrm{kPa}$, and a mainly contractant volumetric behavior is observed. For the dense specimen, the deviatoric stress increases to reach a peak at $q=290 \mathrm{kPa}$ and then a softening regime is observed.
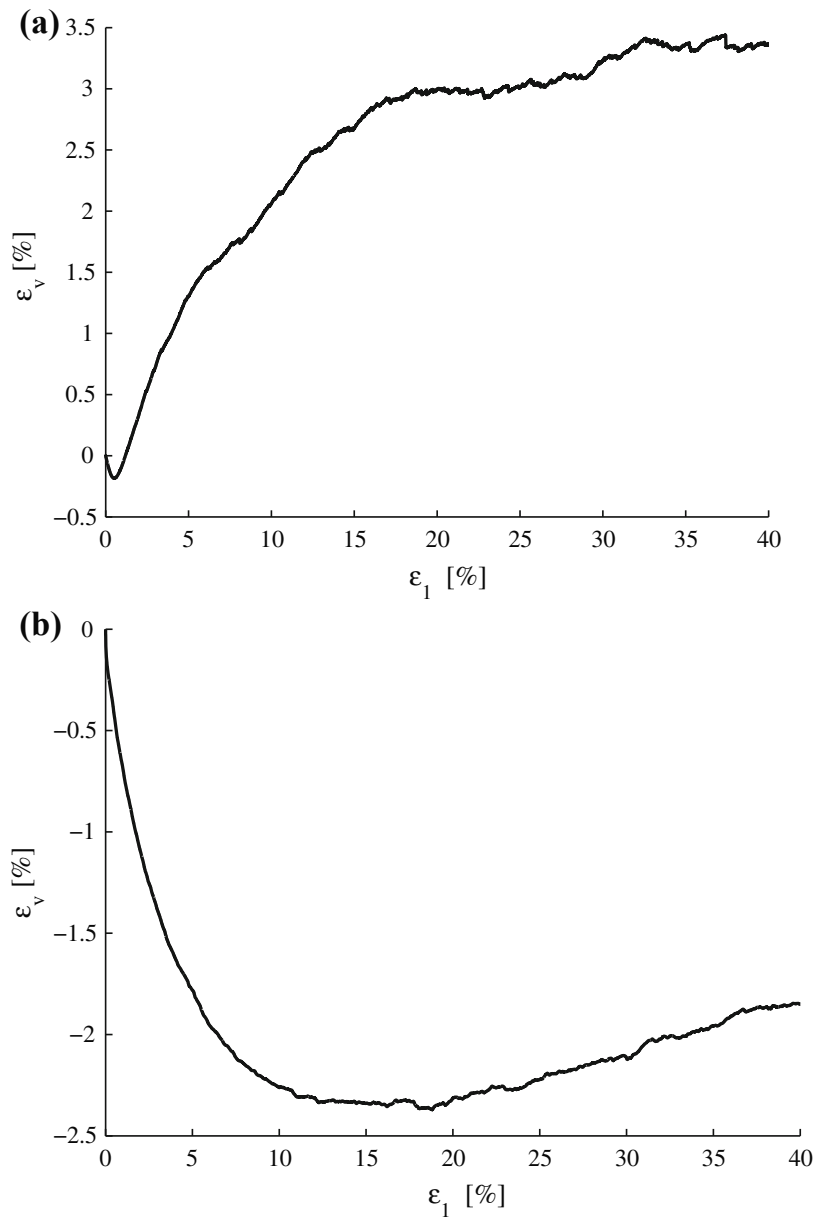

Fig. 3 Volumetric strain in terms of axial strain at a confining pressure of $200 \mathrm{kPa}$ for $S_{1}(\mathbf{a})$, and $100 \mathrm{kPa}$ for $S_{2}$ (b)

\subsection{Second order work from macroscopic variables}

In order to compute the second order work from macroscopic variables, three stress states defined by their deviatoric stress ratio $\eta=\frac{q}{p}$ (where $p$ is the mean pressure in the specimen) are considered (represented by the points $\left(\mathrm{A}_{1}, \mathrm{~B}_{1}, \mathrm{C}_{1}\right)$ and $\left(A_{2}, B_{2}, C_{2}\right)$ in Fig. 2 for dense and loose specimens respectively). These arbitrary stress states are chosen before the Mohr-Coulomb condition is reached and correspond to values of $\eta$ (see Table 2) smaller than $\eta_{\text {peak }}$ for $\mathrm{S}_{1}$ and $\eta_{\text {plateau }}$ for $\mathrm{S}_{2}$ as illustrated in Fig. 2. In particular, $\mathrm{A}_{1}$ and $\mathrm{A}_{2}$ correspond to the isotropic state for each specimen.

The stress states defined above will constitute initial states from which strain probes (as first introduced by Gudehus [7]) 
Table 2 Deviatoric stress ratio $\eta$ corresponding to the stress states $\mathrm{A} 1, \mathrm{~B} 1$ and $\mathrm{C} 1$ for $\mathrm{S} 1$ and $\mathrm{A} 2, \mathrm{~B} 2$ and $\mathrm{C} 2$ for $\mathrm{S} 2$

\begin{tabular}{|c|c|c|c|c|c|c|}
\hline & \multicolumn{3}{|c|}{ Specimen $\mathrm{S}_{1}$} & \multicolumn{3}{|c|}{ Specimen $\mathrm{S}_{2}$} \\
\hline & $\mathrm{A}_{1}$ & $\mathrm{~B}_{1}$ & $\mathrm{C}_{1}$ & $\mathrm{~A}_{2}$ & $\mathrm{~B}_{2}$ & $\mathrm{C}_{2}$ \\
\hline$\eta$ & 0. & 0.76 & 0.95 & 0. & 0.35 & 0.44 \\
\hline
\end{tabular}
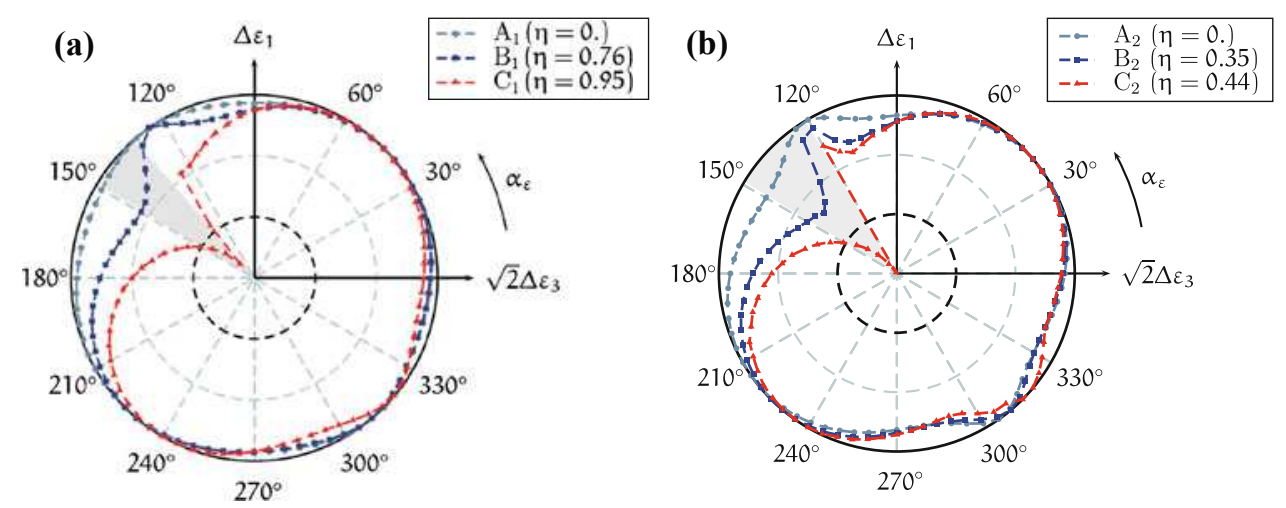

Fig. 4 Circular diagrams of the normalized second order work for dense (a) and loose specimen (b)

are performed. These strain probes consist in a series of strain loading increments $\Delta \bar{\varepsilon}$ defined in the Rendulic strain plane by their direction $\alpha_{\varepsilon}$ (Fig. 2; varying here from $0^{\circ}$ to $360^{\circ}$ with a step of $5^{\circ}$ ) and their norm $\|\Delta \bar{\varepsilon}\|$ (set here equal to $5 \times 10^{-5}$ )

Once the stress response $\Delta \bar{\sigma}$ is computed for each strain loading increment, the macroscopic normalized second order work is computed as $W_{2}^{n}=\Delta \bar{\varepsilon} \cdot \Delta \bar{\sigma} /\|\Delta \bar{\varepsilon}\| \cdot\|\Delta \bar{\sigma}\|[16]$, for all investigated strain directions $\alpha_{\varepsilon}$ and for all considered stress states.

Figure 4 shows circular diagrams of second order work, plotted in the Rendulic plane of strain increments for the initial deviatoric stress ratios corresponding to $\left(\mathrm{A}_{1}, \mathrm{~B}_{1}, \mathrm{C}_{1}\right)$ for $S_{1}$ and $\left(A_{2}, B_{2}, C_{2}\right)$ for $S_{2}$.

For the readability of these representations, a constant $\ell=$ 0.5 is added to the normalized value of $W_{2}$. Consequently, when $W_{2}^{n}$ is negative the plot is inside the dashed circle of radius $r=\ell$, whereas plot is outside the dashed circle for positive values of $W_{2}^{n}$.

Cone of unstable strain directions (illustrated by the gray hatched zone and grouping the incremental strain directions along which the second order work takes negative values) are seen in the second quadrant of the axisymmetric strain increment plane defined by $\left(-\sqrt{2} \Delta \varepsilon_{3}, \Delta \varepsilon_{1}\right)$ at deviatoric stress ratios $\eta=0.95$ and $\eta=0.44$ for $\mathrm{S}_{1}$ and $\mathrm{S}_{2}$ respectively.

\subsection{Second order work from microscopic variables}

The second order work can also be computed according to Eq. (2) from contact forces $\bar{f}^{c}$ and position vector of particles $\bar{x} p$.

To determine the scalar product $\Delta f_{i}^{c} \Delta l_{i}^{c}$ of Eq. (2), increments $\Delta \bar{f}^{c}$ are calculated as the incremental change of the contact force experienced by a given contact during a strain probe in a direction $\alpha_{\varepsilon}$. The branch vector increment $\Delta \bar{l}^{c}$ is deduced from the displacement, during the same strain probing, of particles ' $p$ ' and ' $q$ ' involved in the contact as $\Delta \bar{l}^{c}=\left(\Delta \bar{x}^{p}-\Delta \bar{x}^{q}\right)$. Then summing up scalar products over all the contacts in the specimen, the value of the second order work from microscopic variables $\sum_{p, q} \Delta f_{i}^{c} \Delta l_{i}^{c}$ is computed for a given direction $\alpha_{\varepsilon}$.

The new and lost contacts experienced during the strain probes are also taken into account for the computation of $\Delta f_{i}^{c} \Delta l_{i}^{c}$ where final contact force -for lost contacts- and initial one -for new contacts- are considered as nil.

Figure 5 shows the evolution of the second order work (not normalized) from both macroscopic and microscopic variables at $\eta=0.95$ and $\eta=0.44$ for S1 and S2 respectively in terms of the direction of the strain loading increment $\alpha_{\varepsilon}$.

A good agreement is found between both microscopic and macroscopic expressions of the second order work for the two cases, in the elastic tensorial zone as well as in the plastic tensorial zone ${ }^{1}$ (the zone in light grey on the figure) including the cone of instability (the dashed zone), as well as in the elastic tensorial zone.

Similar results are found for other deviatoric stress states (not displayed here), showing that discrete element method agrees well with the theoretical development.

As a starting point in the microscopic investigation, interest will be focused now on the evolution of the number of

\footnotetext{
1 The plastic tensorial zone groups all directions along which the response to the strain probe has a predominant plastic component com-
} pared to elastic one. 

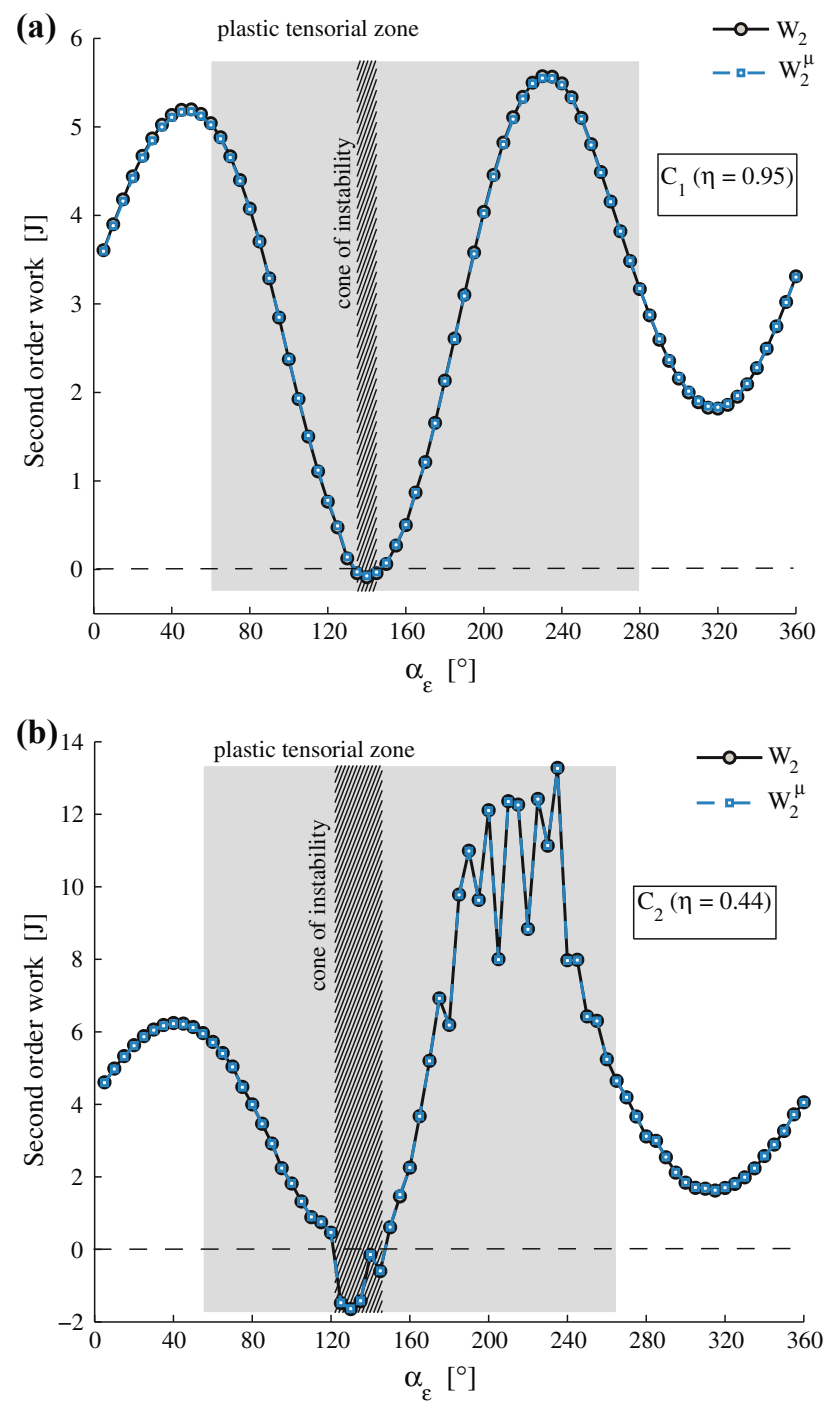

Fig. 5 Comparison between the second order works from both macroscopic and microscopic variables for dense (a) and loose specimen (b)

contacts with negative values of $\Delta f_{i}^{c} \Delta l_{i}^{c}$ along strain probes and their spatial distribution in the specimen.

\section{Spatial distribution of the second order work from microscopic variables}

The aim of this study is to geometrically locate contacts $\left(c^{-}\right)$ defined as contacts with a negative value of $\Delta f_{i}^{c} \Delta l_{i}^{c}$ and characterize their spatial distribution in the specimen. Are these contacts uniformly distributed (individual contact distribution) or gathered together into aggregates separated from each other (grouped contact distribution)?

First and foremost, it is convenient in a preliminary study to make an idea about the evolution of the number $\left(N_{c^{-}}\right)$of contacts $c^{-}$in terms of the direction $\alpha_{\varepsilon}$ of strain probe.

\subsection{Evolution of $\mathrm{N}_{c}^{-}$in the specimen}

The contact network in the specimen constitutes a basic element in the description of microstructure of a granular assembly $[11,12,24,26,28,33]$. The evolution of the total number of contacts $N_{c}$ (scalar term) during lifetime simulation is insufficient to fully describe the contact network, but once related to the mobilized strength of the contact normal force, an improved description may be obtained $[28,33]$ and better conclusions can be drawn on the microscopic behavior. The contact network can be separated into a weak and a strong contact networks with respect to the normal component $f_{n}$ of the contact force. Thus, a contact $c$ belongs to the weak phase if $f_{n}^{c} \leq\left\langle f_{n}^{c}\right\rangle$ and to the strong phase otherwise, where $\left\langle f_{n}^{c}\right\rangle$ is the mean normal force at a given state.

For strain probes with a small increment, even if particle rearrangement occurs in the plastic tensorial zone, a very slight variation in the total number of contacts $N_{c}$ is generally observed, with a very slight increase in the elastic tensorial zone and a very slight decrease in the plastic tensorial zone as illustrated in Fig. 6a, and likewise in Fig. 6b. In this figure, $\Delta N_{c} / N_{c}$ is plotted against the strain probe direction $\alpha_{\varepsilon}$ for the dense specimen at the isotropic stress state $(\eta=0)$ and at a stress state close to the peak of $q(\eta=0.95)$. The fractions of lost and created contacts, compared to the initial total number of contacts existing in the stress state before strain probes also vary slightly around zero. Close to the right border of the tensorial plastic zone, $\Delta N_{c} / N_{c}$ changes its sign from negative to positive whereas no change in sign is observed close to the left border, instead a sharp decrease in $\Delta N_{c} / N_{c}$ is observed. It can be also perceived, that the maximum decrease in the number of contacts (alike in $\Delta N_{c} / N_{c}$ ) does not correspond to directions within the instability cone, but it rather corresponds to strain probe directions around the purely radial extension $\left(\alpha_{\varepsilon}=180^{\circ}\right)$.

Figure 7 shows the evolution of the ratio of contacts belonging to the weak phase $\left(N_{c}^{w} / N_{c}\right)$ and those belonging to the strong phase $\left(N_{c}^{s} / N_{c}\right)$. For both dense and loose specimens, a predominance of the number of contacts $N_{c}^{w}$ involved in the weak phase is observed. Overall, about $60 \%$ (or more) of contacts are carried by the weak contact network whereas only $40 \%$ (or less) are carried by the strong contact network.

Now, we follow the concentration of contacts $c^{-}$for the whole granular assembly by computing the ratio $N_{c^{-}} / N_{c}$, and also for each contact phase respectively, through the values of ratios $N_{c^{-}}^{w} / N_{c}^{w}$ (for the weak phase) and $N_{c^{-}}^{s} / N_{c}^{s}$ (for the strong phase). Results in terms of the strain probing direction are displayed in Fig. 8. For the whole assembly and for both phases, changes in the concentration of $c^{-}$contact is similar, and there is not a qualitative difference in the populating of one of the two contact phases by negative values of second order work at contact. Hence, in all cases there is a 

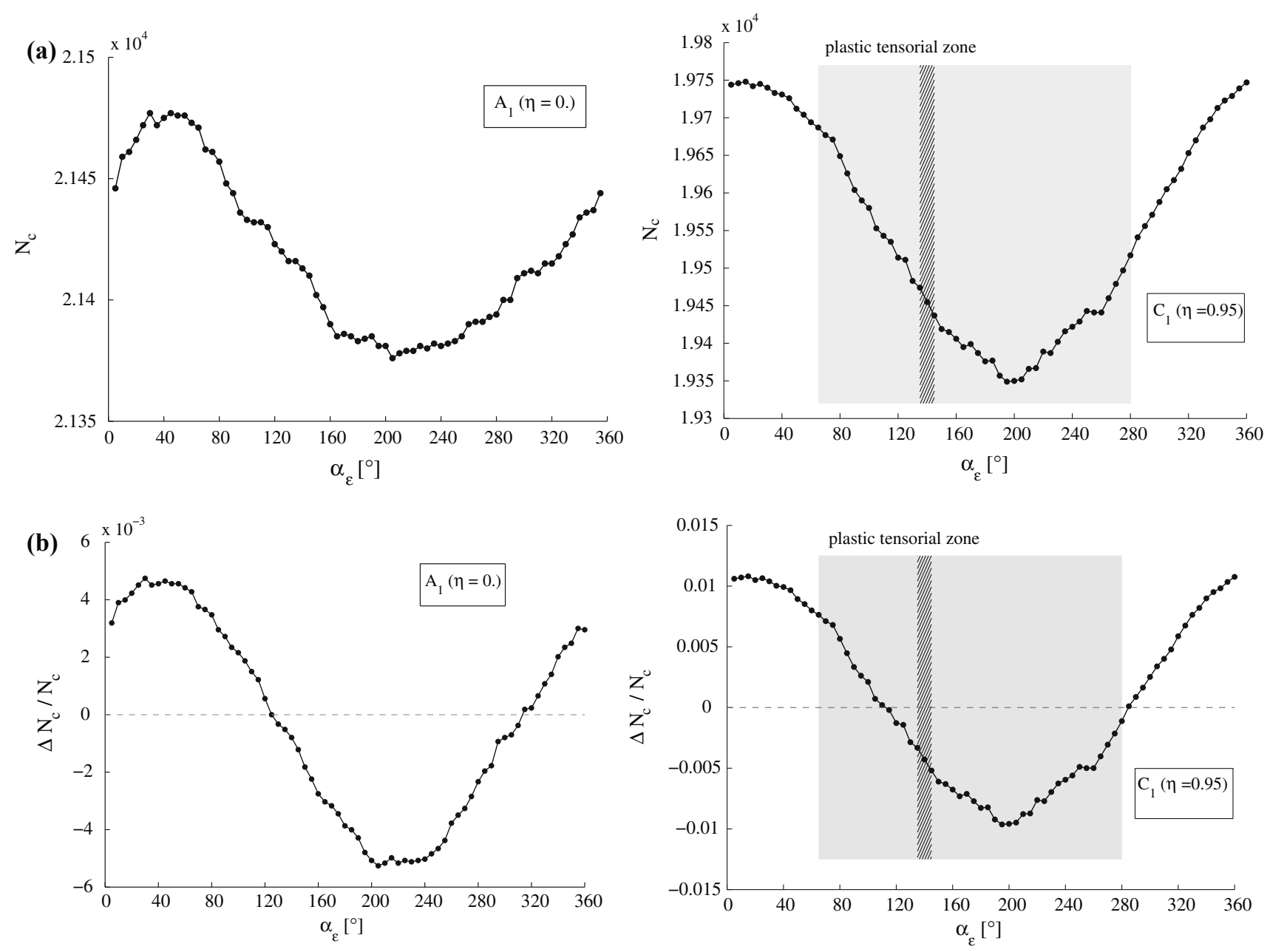

Fig. 6 Evolution of the total contact number $N_{c}$ (a) and $\Delta N_{c} / N_{c}$ (b) in the dense specimen in terms of the strain probe directions at the isotropic stress level and a stress level close to the peak of $q$

strong increase of concentration of $c^{-}$contact in the plastic tensorial zone reaching a peak for directions corresponding to the cone of instability for both specimens. This last result reveals that unstable loading directions whose existence is detected by the vanishing of the second order work computed from macroscopic variables (cone of instability) are deeply related to local mechanisms connected to the vanishing of the second order work defined at contact scale. Moreover, the vanishing of the second order work seems to be more a consequence of the increasing number of $c^{-}$contacts rather than an increase of the amplitude of negative values of the second order work computed from microscopic variables at each contact. This come in line with the quasi-static nature of the simulation where, for the strain loading increments considered here, the particle relative displacements stay limited, and thus amplitudes of contact force increment $\Delta \vec{f}^{c}$ and branch vector increments $\Delta \vec{l}^{c}$ are low.

The main difference between the weak contact phase and the strong phase is quantitative, whatever the loading direction the weak phase is more densely populated by $c^{-}$ contact than the strong phase. For strain directions close to the direction of isotropic compression $\left(\alpha_{\varepsilon}=35.3^{\circ}\right)$ the ratio $N_{c^{-}}^{s} / N_{c}^{s}$ for the strong phase is even almost nil, and only the weak phase keep a small proportion of contacts presenting a negative second order work.

In particular the weak phase may become the quasi unique contributor at the borders of the plastic tensorial zone, especially for the dense specimen. Contribution of the strong phase, carrying the whole deviatoric load [29], becomes particularly visible in the plastic tensorial zone where this phase is essential to insure the stability of the specimen. Inversely, the weak phase able to balance an isotropic pressure [29] seems to play a key role for strain loading directions around the direction relative to the isotropic compression and is the only contributor. However, the highest contribution for both phases (i.e. highest values of $N_{c^{-}}^{w} / N_{c}^{w}$ and $N_{c^{-}}^{s} / N_{c}^{s}$ and consequently highest value of $N_{c^{-}} / N_{c}$ ) matches with the strain directions belonging to the cone of instability. Hence, both 

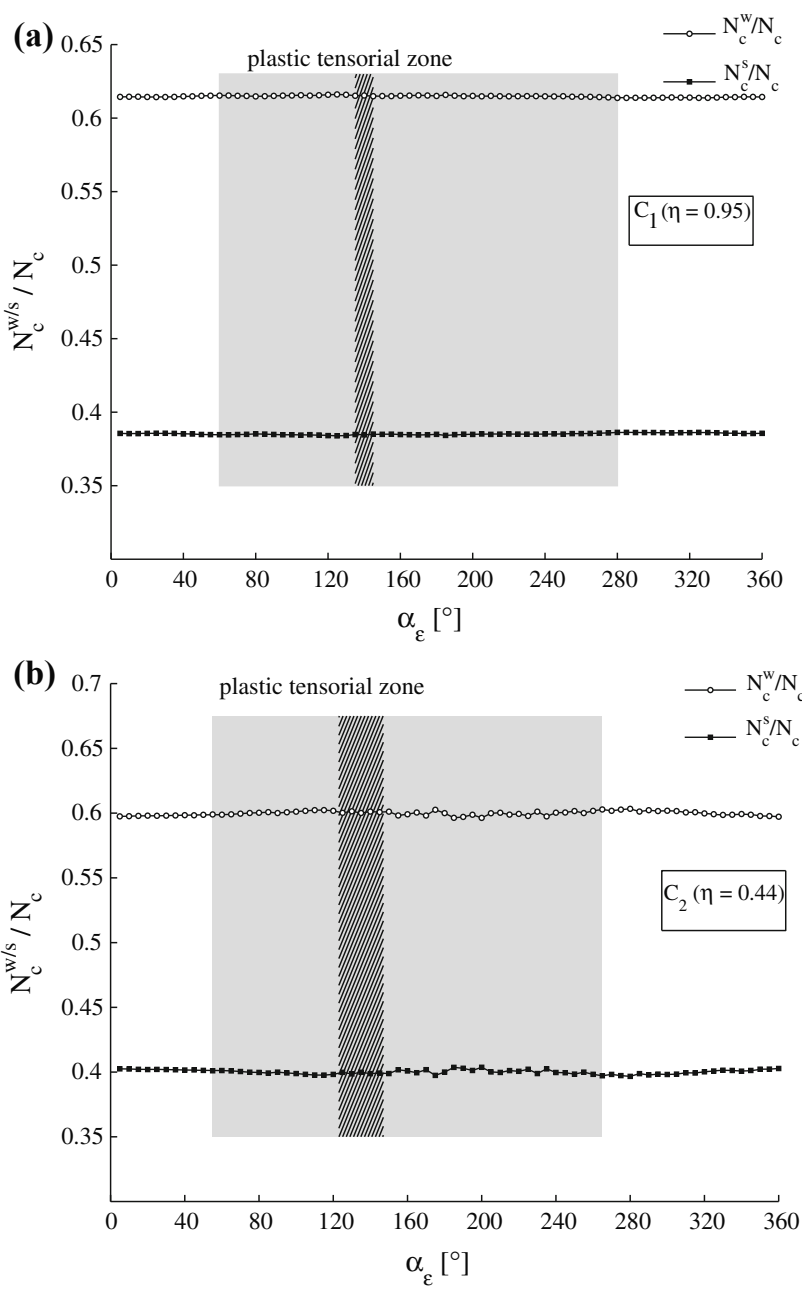

Fig. 7 Evolution of the contact number according to its belonging to weak and strong contact networks with respect to the total number of contact, in terms of the strain probe direction for dense (a) and loose specimen (b)

contact networks are involved in the vanishing of the macroscopic second order work but with a weight more important of the weak contact network whatever the strain loading direction.

A second study is considered herein in order to confirm the previous results and to show which phase (among the weak and strong phases) contributes the most in the production of $c^{-}$contacts. For this purpose, the number of $c^{-}$contacts produced by each phase is compared to the total number of $c^{-}$contacts, namely the ratios $N_{c^{-}}^{s} / N_{c^{-}}$and $N_{c^{-}}^{w} / N_{c^{-}}$. The evolution of these two quantities, along with $N_{c}^{s} / N_{c}$ and $N_{c}^{w} / N_{c}$ (already plotted separately in Fig. 7), in terms of the strain probe direction $\alpha_{\varepsilon}$ is plotted in Fig. 9 for both dense and loose specimens at stress states close to the peak and to the plateau of $q$ respectively. It can be concluded that the weak phase produces the major part of $c^{-}$contacts. This comes in line with the previous conclusion since the concentration in
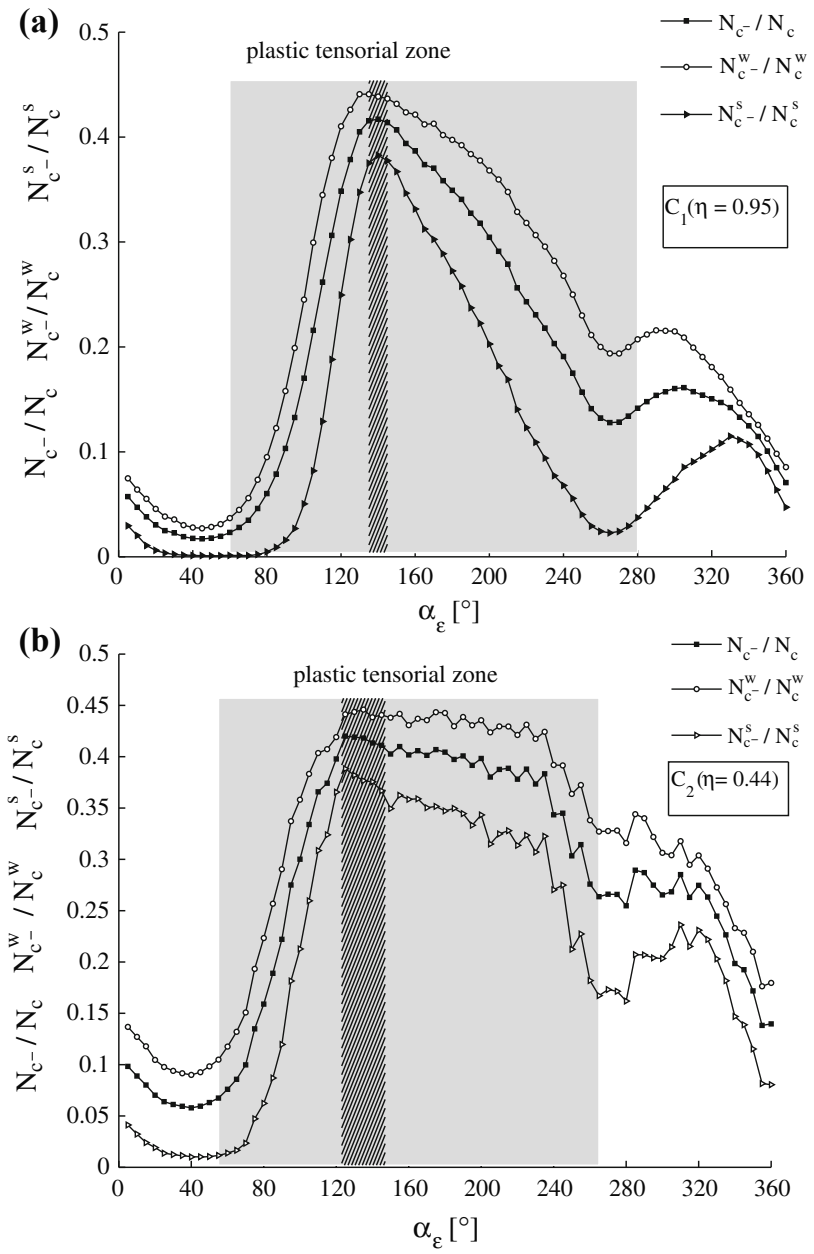

Fig. 8 Evolution of the concentrations of $c^{-}$contacts in the whole specimen, in the weak and strong contact networks respectively, in terms of the strain probe direction for the dense (a) and loose specimen (b)

$c^{-}$was found to be more important in the weak phase, which has the largest number of contacts.

Moreover, Fig. 9 points out another interesting point; the directions along which the second order work (computed from macroscopic variables) vanishes (cone of unstable directions) are characterized by a sharp increase in the production of $c^{-}$contacts in the strong phase accompanied by a similar decrease of the weak phase participation.

In contrast, the contribution of the strong phase in the production of $c^{-}$contacts is lower along the directions for which the second order work has positive values

Figures 8 and 9 confirm that the weak phase is more subjected to vanishing microscopic second order works.

The weak phase acts as a confining and stabilizing structure with respect to the strong phase, limiting the collapse of the force chains and insuring the load bearing capacity of the granular assembly [33,37]. As the weak phase is more densely populated with $c^{-}$contacts, we may imagine that the loss of bearing capacity of a specimen in the case of 

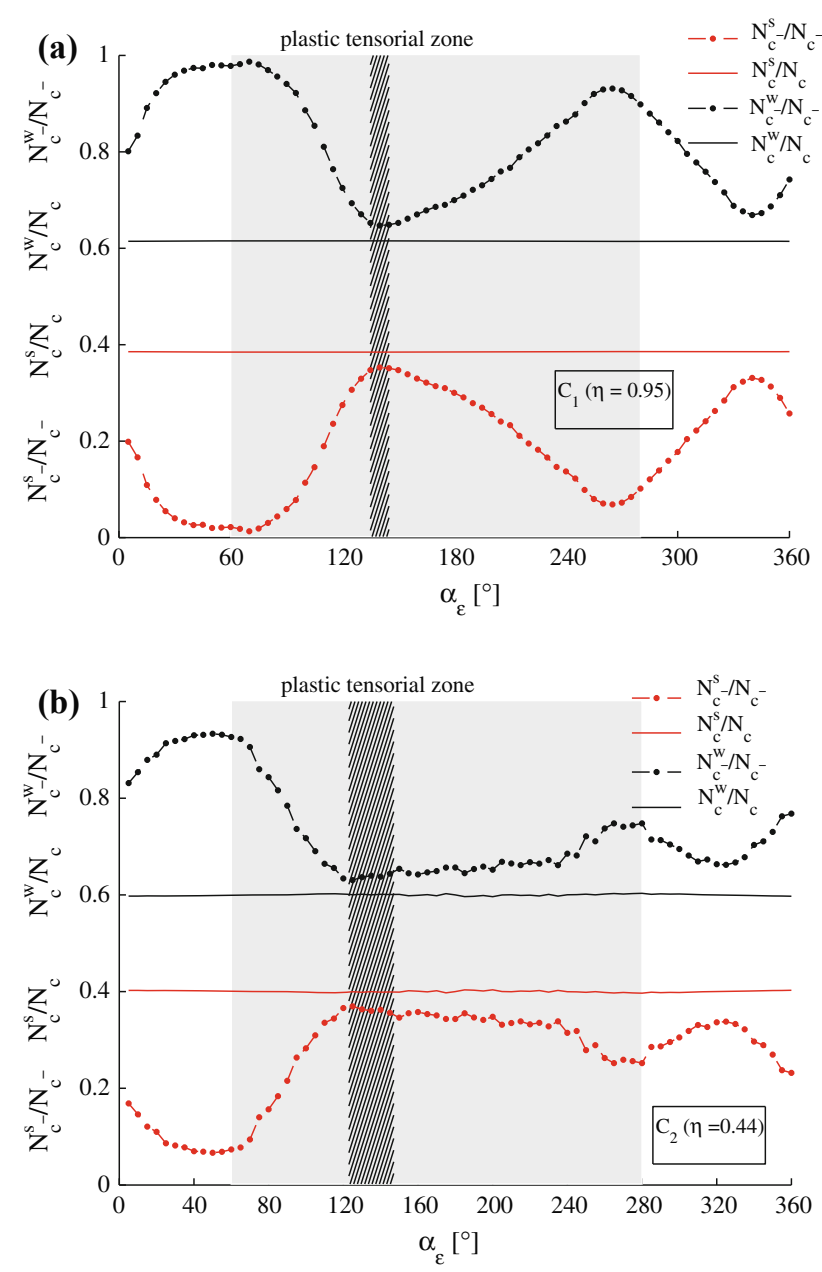

Fig. 9 Evolution of $N_{c^{-}}^{s} / N_{c^{-}}, N_{c}^{s} / N_{c}, N_{c^{-}}^{w} / N_{c^{-}}$and $N_{c}^{w} / N_{c}$ in terms of the strain probe direction for dense (a) and loose specimen (b)

failure development from an unstable state, is related to a reduction of the confining and stabilizing role of the weak phase offering the possibility for the force chains to collapse (characterized by a strong increase of the $c^{-}$population in the strong phase).

The previous study shows that both strong and weak phases are occupied by $c^{-}$but this cannot fully confirm the homogeneity of the geometric spatial distribution of $c^{-}$in the specimen.

\subsection{Homogeneity of the distribution of contacts \\ $c^{-}$in the specimen}

As illustrated in Fig. 10, an elementary cubic volume with variable edge length $l \leq L$ initially centered in the specimen is considered in order to compute the number of contacts $N_{c^{-}}$with negative values of $\Delta f_{i}^{c} \Delta l_{i}^{c}$ in this expanding cube. $l$ is set equal to $2 D_{S}$ (where $D_{s}$ is the mean particle diameter of the granular assembly) for the initial volume, and then

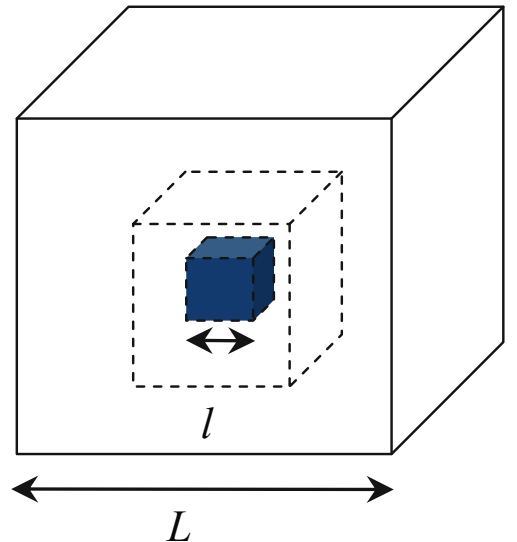

Fig. 10 Illustration of the cubic elementary volume variation
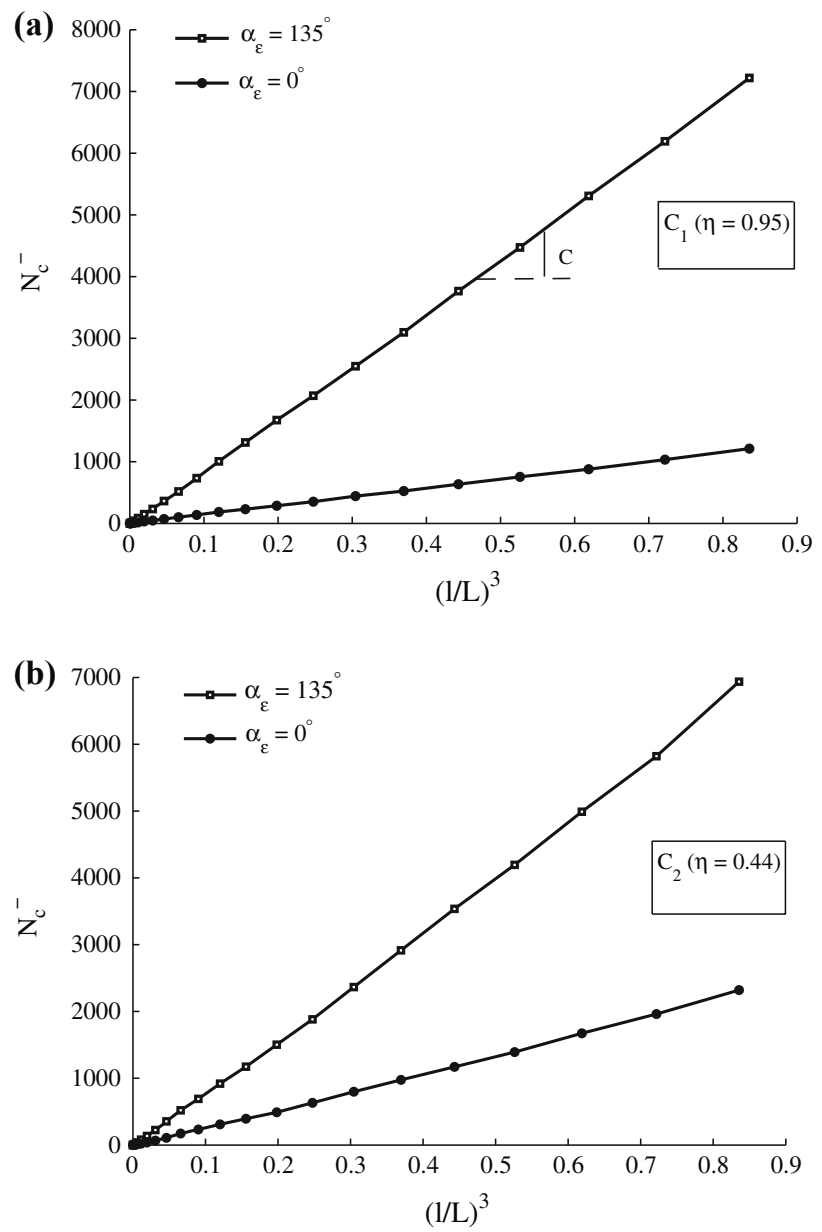

Fig. 11 Evolution of $N_{c^{-}}$in terms of $(l / L)^{3}$ for dense (a) and loose specimen (b)

gradually increased by steps of $2 D_{S}$ until the boundaries of the specimen are reached $(l=L)$.

The evolution of $N_{c^{-}}$in terms of $(l / L)^{3}$ is reported in Fig. 11 for dense specimen at $\eta=0.95$ and for loose 
Table 3 Results of the $\chi^{2}$ goodness of fit test evaluating the homogeneity of $c^{-}$contacts spatial distribution for dense (S1) and loose (S2) specimens after performing strain probes into stable $\left(\alpha_{\varepsilon}=0^{\circ}\right)$ and unstable $\left(\alpha_{\varepsilon}=135^{\circ}\right)$ directions

\begin{tabular}{|c|c|c|c|c|}
\hline Specimen & S1 & & S2 & \\
\hline Strain probe direction & $\alpha_{\varepsilon}=0^{\circ}$ & $\alpha_{\varepsilon}=135^{\circ}$ & $\alpha_{\varepsilon}=0^{\circ}$ & $\alpha_{\varepsilon}=135^{\circ}$ \\
\hline$H_{0}$ Hypothesis of the $\chi^{2}$ test & Rejected & Not rejected & Rejected & Not rejected \\
\hline
\end{tabular}

The H0 hypothesis of the $\chi^{2}$ test, corresponding to the hypothesis of a homogeneous distribution of $c^{-}$contacts, was tested with a significance level set at $5 \%$
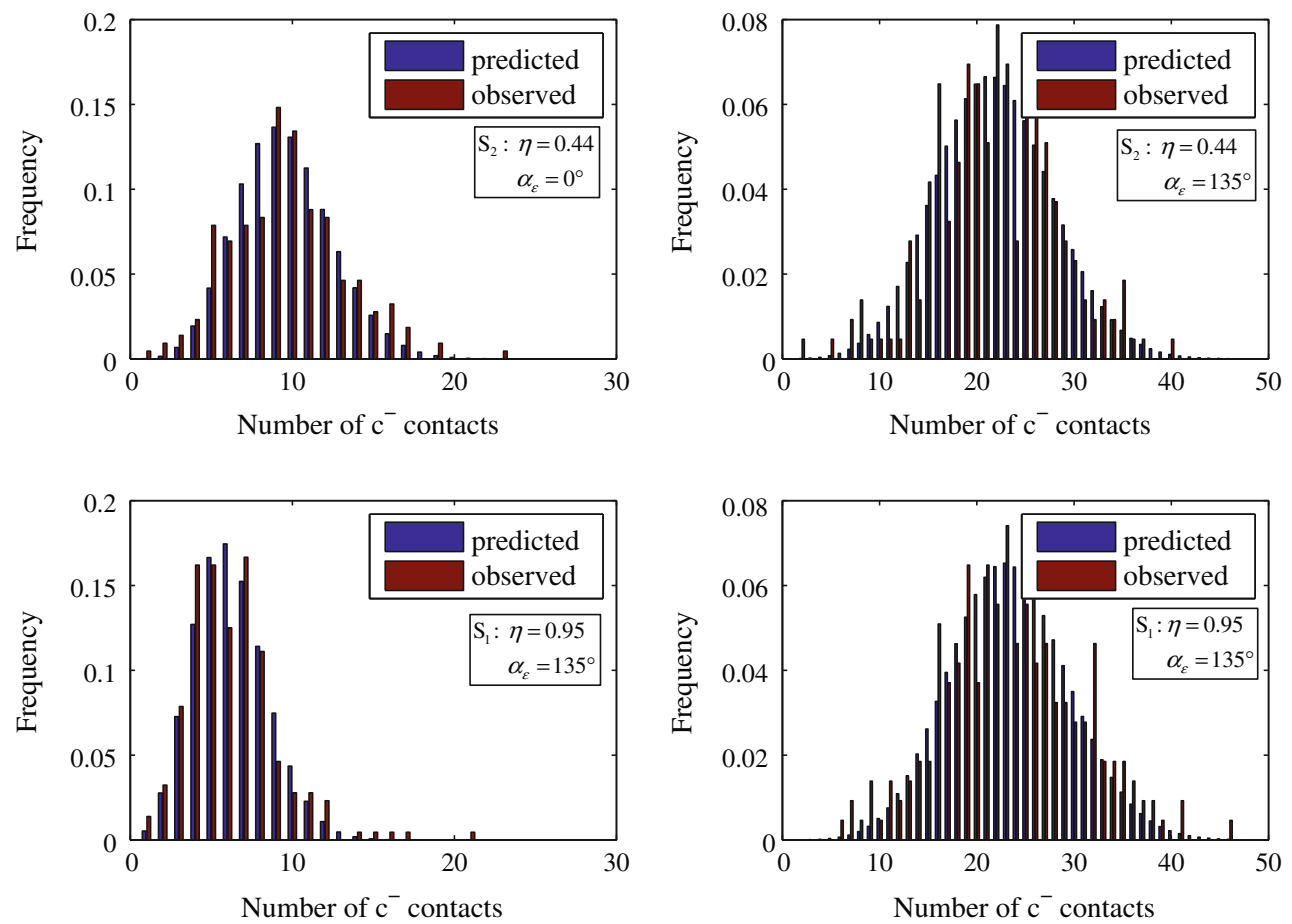

Fig. 12 Comparison between the observed distributions and the corresponding theoretical Poisson distributions of the number of $c^{-}$contacts in the cubic sub-volumes for dense and loose samples at $\eta=0.95$ and

$\eta=0.44$ respectively and for two strain probe directions corresponding to a stable direction $\left(\alpha_{\varepsilon}=0^{\circ}\right)$ and to an unstable one $\left(\alpha_{\varepsilon}=135^{\circ}\right)$

specimen at $\eta=0.44 . N_{c^{-}}$increases linearly for all cases and in all directions of strain probes (for simplicity and clarity reasons, only two directions are shown: $\alpha_{\varepsilon}=0^{\circ}$ (stable direction corresponding to a lateral extension) and $\alpha_{\varepsilon}=135^{\circ}$ (direction in instability cone).

It can be noticed that $N_{c^{-}}$is deeply related to the direction of strain probe, it jumps considerably to a value almost three times bigger for the loose specimen and eight times bigger for the dense specimen from a stable direction $\left(\alpha_{\varepsilon}=0^{\circ}\right)$ to an unstable one $\left(\alpha_{\varepsilon}=135^{\circ}\right)$.

These linear like curves shows also that the geometrical distribution of $c^{-}$in the specimen is almost homogeneous, since $N_{c^{-}}$is proportional to the dimensionless quantity $(l / L)^{3}$ such that $N_{c^{-}}=C(l / L)^{3}$, where $C$ is the slope of curve and defines the volumic concentration of $N_{c^{-}}$.

The homogeneity of the spatial distribution of $c^{-}$in the specimen can also be evaluated using a statistical analysis of the distribution of the number of $c^{-}$contacts in sub-volumes of the specimen. The repartition of $c^{-}$contacts follows a Poisson point process if no spatial structure of the $c^{-}$contacts exists, (i.e. $c^{-}$contacts are homogeneously distributed) [4]. Thus, if the sample is divided into $n$ sub-volumes of equal volume $V_{n}$, the distribution of the number $n_{c^{-}}$of $c^{-}$ contacts in the sub-volumes can be computed. The probability $P\left(n_{c^{-}}=k\right)$ for having $k \quad c^{-}$contacts into a sub-volume can therefore be expressed as follows:

$P\left(n_{c^{-}}=k\right)=\frac{\lambda^{k} e^{-\lambda}}{k !}$

where $\lambda$ can be estimated by the mean likelihood $E\left(n_{c^{-}}\right)$ of the $c^{-}$contacts in the different elementary volumes. 
In our context, the specimen is divided into 216 cubical subvolumes with an edge length of $4 D_{s}$ [39]. The number $n_{c^{-}}$ of $c^{-}$contacts in each sub-volume is then calculated. The observed distribution of $n_{c^{-}}$is compared with the theoretical corresponding Poisson distribution with the parameter $\lambda=E\left(n_{c^{-}}\right)$.

The $\chi^{2}$ goodness of fit test $[15,27,32]$ was used to test whether the theoretical Poisson distribution matches well of the observed distribution of $n_{c^{-}}$in the sub-volumes.

The statistical analysis exposed above is used to evaluate the homogeneity of the dense and loose specimens at $\eta=0.95$ and $\eta=0.44$ respectively after performing strain probes into two directions corresponding to a stable direction $\left(\alpha_{\varepsilon}=0^{\circ}\right)$ and to an unstable one $\left(\alpha_{\varepsilon}=135^{\circ}\right)$.

The results from the $\chi^{2}$ goodness of fit test (Table. 3) show that the distribution of $n_{c^{-}}$follows a Poisson distribution from both dense and loose samples in the unstable direction whereas it is not the case in the stable direction. The statistical tests therefore highlight that the distribution of $c^{-}$contacts is homogeneous for both loose and dense specimens into the unstable direction. On the contrary, for stable directions, the statistical tests do not allow concluding for the homogeneity of the distribution of $c^{-}$contacts. However, the qualitative adequacy of the global shape of the observed distribution and the corresponding theoretical Poisson distribution of $n_{c^{-}}$(Fig. 12) tends to show that the spatial distribution of $c^{-}$contacts is almost homogeneous, i.e. that no significant clustering pattern exists. These results have been confirmed for different sizes of the cubic sub-volumes with or without considering the sub-volumes at the edges of the specimens.

The significant tendency in the homogeneity of the $c^{-}$ contacts in the specimen confirms the results observed for the elementary cubic volumes with variable edges. As $c^{-}$contacts are almost homogeneously distributed in the specimen, the concentration $C$ of $N_{c^{-}}$can be used as a representative indicator of the proportion of $c^{-}$contacts in the specimen.

The concentration $C$ of $N_{c^{-}}$can be plotted in terms of both strain probe direction $\alpha_{\varepsilon}$ and stress ratio $\eta$ as shown in Fig. 13. The variation of $C$ in terms of $\alpha_{\varepsilon}$ reaches its highest values in the plastic tensorial zone, along directions within the cone of instability where the second order work vanishes. A tight correspondence between the minima and maxima of $C$ and $W_{2}$ (Fig. 5) is also distinguished, the minima of $W_{2}$ corresponds to the maxima of $C$ and vice-versa.

It is also well illustrated that the volumetric concentration $C$ increases along directions within to the cone of instability and when the deviatoric stress state defined by $\eta$ gets closer to the peak/plateau of the $q=f\left(\varepsilon_{1}\right)$ curve.

Two main results can be put forward from the previous study. First, the contacts with negative values of $\Delta f_{i}^{c} \Delta l_{i}^{c}$ are almost homogeneously distributed in the specimen although statistical analysis shows a significant homogeneity of $c^{-}$
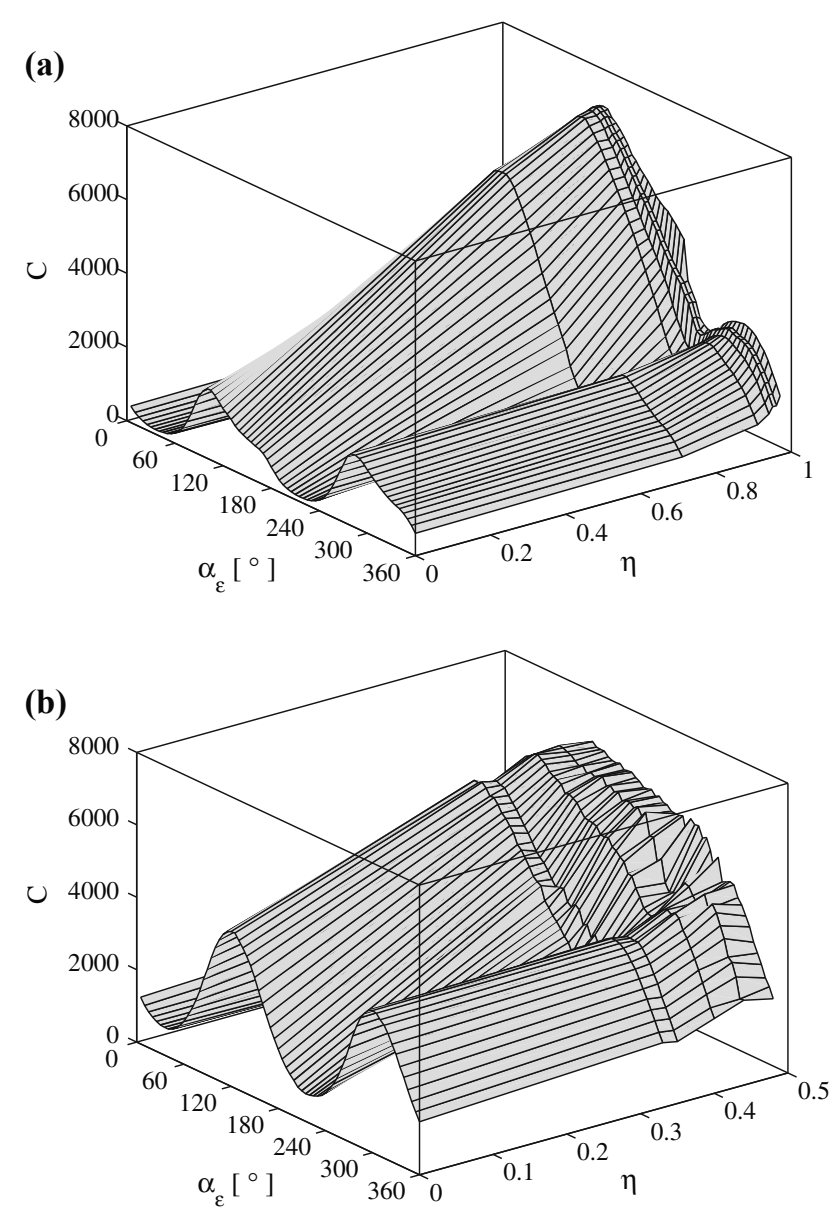

Fig. 13 Evolution of $\mathrm{C}$ in terms of both stress ratio and strain probe direction for dense (a) and loose specimen (b)

contacts along unstable directions and a slight tendency towards non homogeneity of $c^{-}$contacts for stable directions. Second, $N_{c^{-}}$depends strongly on the deviatoric stress state and on the loading direction.

These results provide a solid platform for the next investigation in the same context concerning the existence of any correlation length between contacts with negative values of $\Delta f_{i}^{c} \Delta l_{i}^{c}$.

\subsection{Minimal distance between the contacts $\mathrm{c}^{-}$}

In order to check whether the positions of contacts $c^{-}$are uniform (individual contact distribution) or gathered together into aggregates (grouped contact distribution), another analysis is considered. The principle consists in computing for each contact $c_{k}^{-}$in the specimen, with $k=\left\{1, \ldots, N_{c^{-}}\right\}$, the minimum distance $d_{\min }^{k}$ between this contact and the nearest contact $c_{m}^{-}(m \neq k)$ in the neighborhood. The contact $c_{k}^{-}$and the corresponding computed distance $d_{\min }^{k}$ are then stored into a list. In a first study, we focus on the mean value $\left\langle d_{\min }\right\rangle$ of all computed distances $\left\{d_{\min }^{1}, \ldots, d_{\min }^{N_{c^{-}}}\right\}$. 

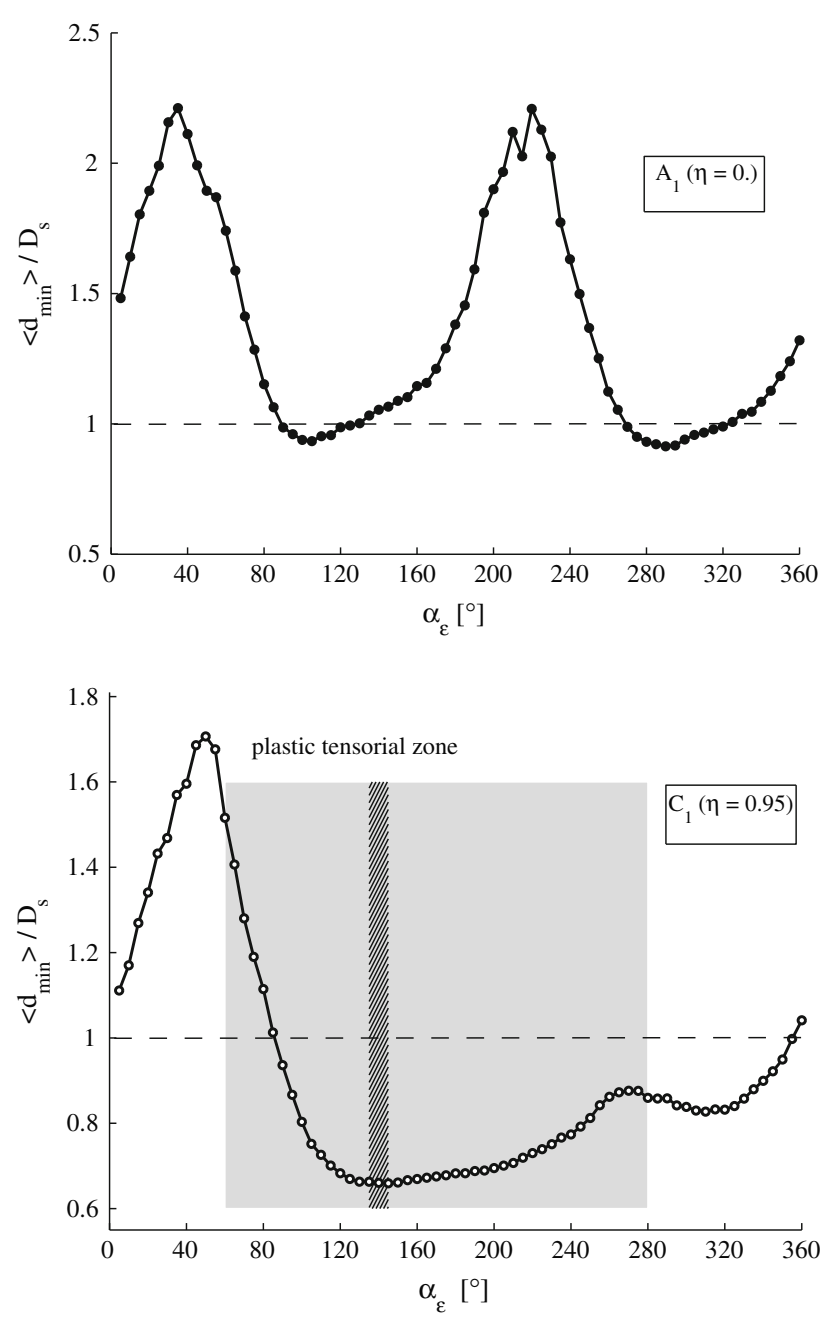

Fig. 14 Evolution of $\left\langle d_{\min }\right\rangle / D_{s}$ in terms of strain probe directions for the dense specimen at different stress ratio

The evolution of $\left\langle d_{\min }\right\rangle$ normalized to $D_{s}$ in terms of $\alpha_{\varepsilon}$ for the dense specimen is plotted in Fig. 14 for $\eta=0$ and $\eta=0.95$ respectively.

The smallest values of $\left\langle d_{\min }\right\rangle$ correspond to the directions in the cone of instability, $\left\langle d_{\min }\right\rangle$ decreases as long as the deviatoric stress state approaches the peak/plateau or $\alpha_{\varepsilon}$ gets inside or closer to the cone of instability. In addition, the same correspondence between the minima and maxima of $\left\langle d_{\min }\right\rangle / D_{s}$ and $W_{2}$ (Fig. 5) is observed which finally comes in line with the previous study since $N_{c^{-}}$increases in these directions and the distances between $c^{-}$shrinks.

In a second study, the same reasoning is applied in order to detect agglomerations of contacts $c^{-}$(if any) such as doublet, triplet. .., but instead of storing the minimal distance $d_{\min }^{k}$ between $c_{k}^{-}$and $c_{m}^{-}$in the neighborhood, the twenty first minimal distances are considered. Thus for each contact $c_{k}^{-}$ twenty values $d_{\min _{i}}^{k} i=(0, \ldots, 20)$ are stored into a list as follows

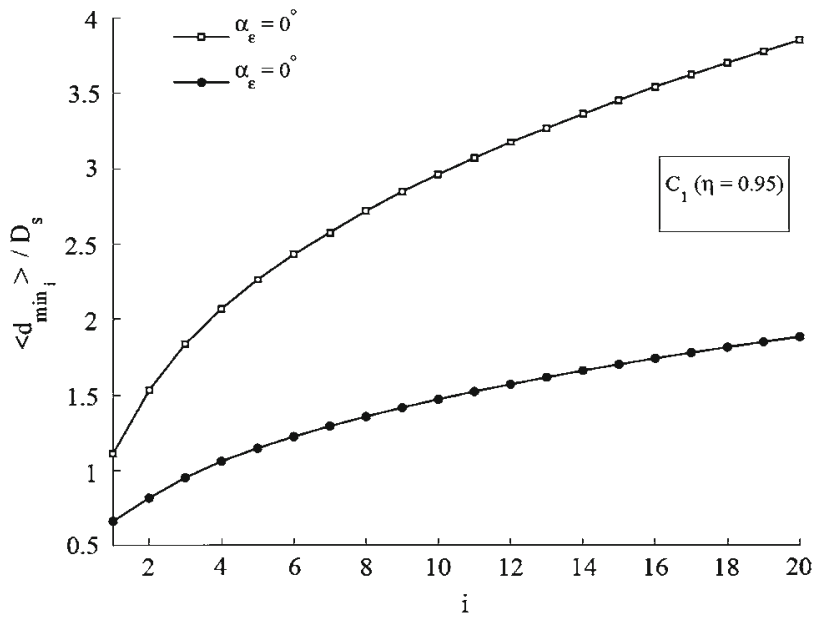

Fig. 15 Evolution of $\left\langle d_{\min _{i}}\right\rangle$ in terms of $i$, dense specimen

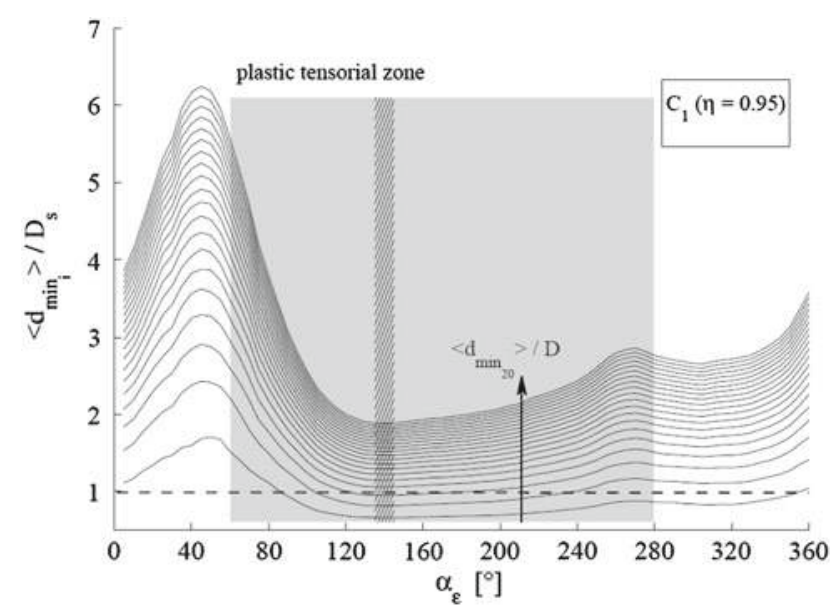

Fig. 16 Evolution of the twenty $\left\langle d_{\min _{i}}\right\rangle$ in terms of the strain probe direction for the dense specimen

$$
\begin{aligned}
& \left\{\begin{array}{c}
c_{1}^{-} \\
c_{2}^{-} \\
\cdot \\
\cdot \\
c_{N_{c^{-}}}^{-}
\end{array}\right\}\left\{\begin{array}{cccc}
d_{\min _{1}}^{1} & d_{\min _{2}}^{1} & \cdots & d_{\min _{20}}^{1} \\
d_{\min _{1}}^{2} & d_{\min _{2}}^{2} & \cdots & d_{\min _{20}}^{2} \\
\cdot & \cdot & \cdots & \cdot \\
\cdot & \cdot & \cdots & \cdot \\
d_{\min _{1}}^{N_{c^{-}}} & d_{\min _{2}}^{N_{c^{-}}} & \cdots & d_{\min _{20}}^{N_{c^{-}}}
\end{array}\right\} \\
& \left\langle d_{\min _{1}}\right\rangle\left\langle d_{\min _{2}}\right\rangle \cdots\left\langle d_{\min _{20}}\right\rangle
\end{aligned}
$$

Figure 15 shows that $\left\langle d_{\min _{i}}\right\rangle$ increases smoothly in terms of the rank $i$ with no sharp variations that could reflect a tendency or inclination toward a specific gathering to which the contacts $c^{-}$may be subjected.

Figure 16 shows, for the twenty considered minimal distances, that the evolution of $\left\langle d_{\min _{i}}\right\rangle$ in terms of $\alpha_{\varepsilon}$ is identical to the first minimal distance $\left\langle d_{\min _{1}}\right\rangle$, with lowest values in the tensorial plastic zone and more precisely within the cone of instability. The curves representing $\left\langle d_{\min _{i}}\right\rangle$ keep the same shape independently of the rank $i$ which proves that for 

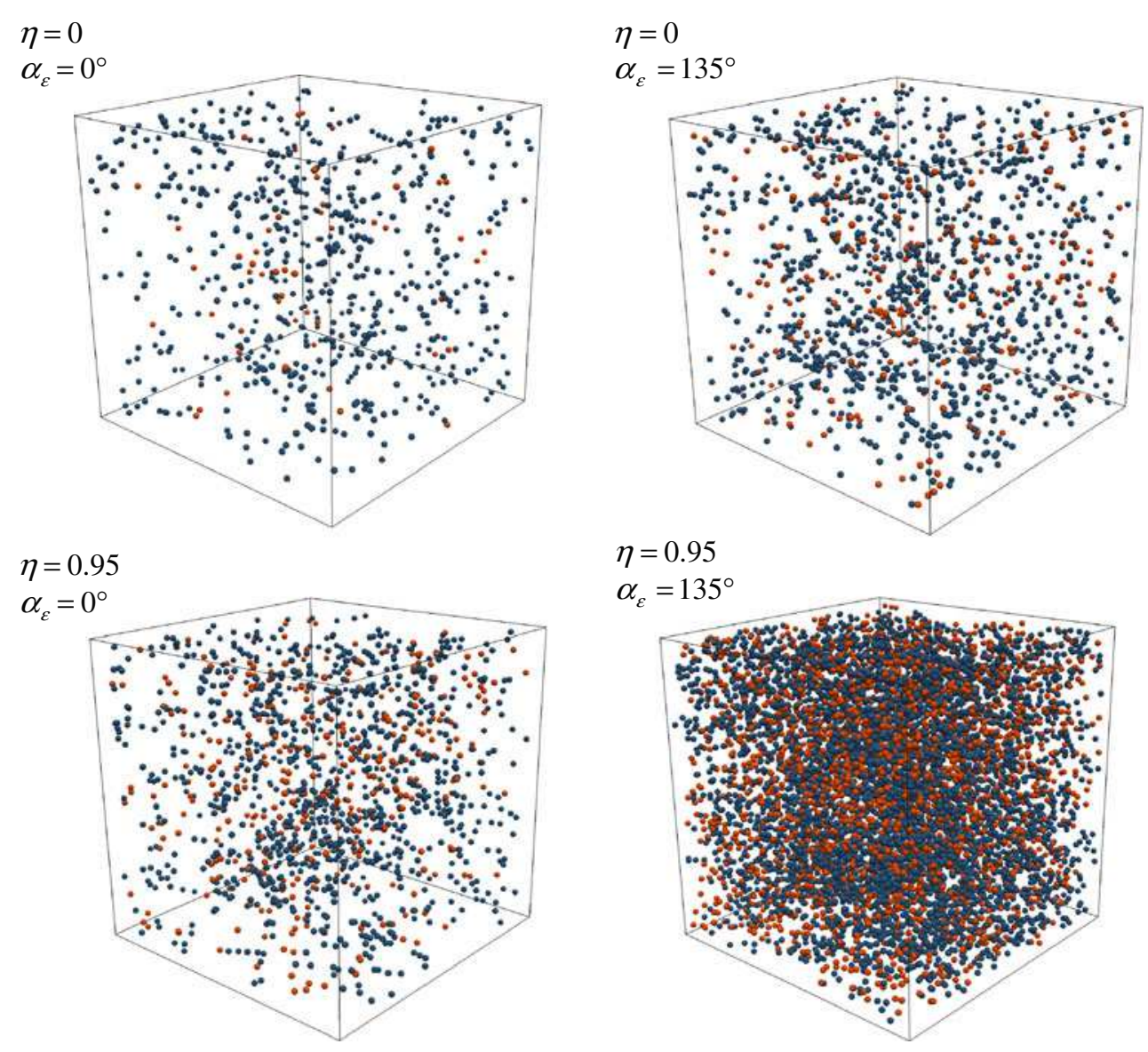

Fig. 17 Three dimensional views of contacts with negative second order work according to their belonging to strong (light) and weak (dark) contact networks for the dense specimen

each strain probing direction, the contacts $c^{-}$have almost the same neighborhood distribution on average in the spherical volume of radius $R=\bar{d}_{\min _{20}}$ which can reach up to $27 \%$ (about $6.25 \times D_{S}$ ) of the specimen edge's length for strain probes directions within the elastic tensorial zone; and $8.6 \%$ (about $2 \times D_{S}$ ) of the specimen edge's length for strain probes directions within the cone of instability.

Finally, a three-dimensional graphical representation of contacts with negative values of second order work from microscopic variables is proposed as an illustration of the analysis reported above (see Fig. 17).

The light spheres represent the contacts $c^{-}$held by the strong network, whereas the dark ones belong to the weak contact network. Boundary effects may be noticed, but expected as well since the walls are frictionless and the vanishing the microscopic second order work for a purely elastic wall-particle contact cannot occur.

The 3D representations confirm both results quantitatively shown in the previous study; first, the homogeneity of the spatial distribution of contacts $c^{-}$within the specimen; and second, the competition between strong and weak phase in holding contacts with negative values of microscopic second work with a predominance of the weak phase.

From this study, the neighborhood (in terms of spatial distribution of contacts $c^{-}$) of $c^{-}$seems to not be qualitatively affected by the strain loading direction. There is only a quantitative dependence between the density of contacts $c^{-}$homogeneously spread within the sample and the strain loading direction; with the particular case of unstable strain direction grouped within the cone of unstable directions for which the $c^{-}$density is the highest.

\section{Concluding remarks}

On the basis of a discrete numerical model, relation between unstable loading directions (characterized by negative values of the macroscopic expression of the second order work) and the elementary terms constituting the microscopic expression of the second order work (i.e. $\Delta f_{i}^{c} \Delta l_{i}^{c}$ ) have been investigated. 
Firstly, it was confirmed from a numerical point of view that both microscopic and macroscopic expressions of second order work are equivalent over a representative elementary volume, in a quasi-static regime of deformation. Then, focus was concentrated on the spatial distribution, throughout the granular assembly, of the contacts named $c^{-}$, bringing a negative contribution to the second order work. It was shown that both weak and strong contact networks are populated by such contacts and thus contribute to the vanishing of the second order work at the macroscopic scale. However, in all cases the weak phase is more densely populated and is consequently the most important contributor of $c^{-}$contacts. A good agreement is found between the unstable loading directions (forming the cone of instability) and the density of population of $c^{-}$contacts, which is, for these directions, the highest in the weak and the strong contact networks. Hence, the development of unstable loading directions seems more likely related to a multiplication of $c^{-}$contacts (i.e. of potentially unstable contacts) rather than an increase of the contribution of some pre-existing $c^{-}$contacts.

The loading directions included inside the cone of instability are leading to a sudden effective failure as soon as a proper control variable is chosen for this loading, according to second order work criterion and the three necessary and sufficient conditions for failure [18,22]. It has been shown in this paper that the distribution of unstable intergranular contacts is uniform for these directions. It was shown that $c^{-}$contacts are merely homogeneously distributed within the sample. No significant aggregate structure (grouped contact) was identified even though the statistical analysis of the distribution of $c^{-}$contacts shows a slight tendency towards non homogeneity for stable directions. Thus, the failure mode which would be induced from the considered stress-strain states by a proper control parameter will be also homogeneous, corresponding to what has been called a "diffuse" failure mode [23]. Analyzing the causes of this potential non homogeneity for stable directions constitutes an interesting perspective for further research works.

Finally, as the weak contact network is the most densely populated with $c^{-}$contacts we may imagine that, due to this important population of potentially unstable contacts, the weak phase could present some defects in its role of propping-up the force chains carrying the deviatoric loading. Affected by this loss of support, some force chains could present an unstable configuration resulting, at the scale of the granular assembly, in an unstable mechanical response when it is loaded in a direction included in the cone of instability. However, this last point is still an open question and further micromechanical investigations are necessary to better understand the relation between the $c^{-}$contacts, the unstable mechanical response and finally failure of the granular assembly.

\section{References}

1. Antony, S., Momoh, R., Kuhn, M.: Micromechanical modelling of oval particulates subjected to bi-axial compression. Comput. Mater. Sci. 29(4), 494-498 (2004)

2. Bagi, K.: Analysis of microstructural strain tensors for granular assemblies. Int. J. Solids Struct. 43(10), 3166-3184 (2006)

3. Cundall, P.-A., Strack, O.-D.-L.: A discrete numerical model for granular assemblies. Geotechnique 29(1), 47-65 (1979)

4. Diggle, P.J.: Statistical Analysis of Spatial Point Patterns, p. 148. Academic press, London (1983)

5. Gardiner, B., Tordesillas, A.: Micromechanics of shear bands. Int. J. Solids Struct. 41(21), 5885-5901 (2004)

6. Golshani, A., Okui, Y., Oda, M., Takemura, T.: A micromechanical model for brittle failure of rock and its relation to crack growth observed in triaxial compression tests of granite. Mech. Mater. 38(4), 287-303 (2006)

7. Gudehus, G.: A comparison of some constitutive laws under radially symmetric loading and unloading. In: Wittke, I.W. (ed.) 3rd International Conference on Numerical Methods in Geomechanics, pp. 1309-1323. A.A. Balkema, Aachen (1979)

8. Hill, R.: A general theory of uniqueness and stability in elasticplastic solids. J. Mech. Phys. Solids 6, 236-249 (1958)

9. Iwashita, K., Oda, M.: Micro-deformation mechanism of shear banding process based on modified distinct element method. Powder Technol. 109(1-3), 192-205 (2000)

10. Kuhn, M.R.: Structured deformation in granular materials. Mech. Mater. 31, 408-429 (1999)

11. Kuhn, M.R.: Micro-mechanics of fabric and failure in granular materials. Mech. Mater. 42(9), 827-840 (2010)

12. Kuhn, M.R., Bagi, K.: Contact rolling and deformation in granular media. Int. J. Solids Struct. 41(21), 5793-5820 (2004)

13. Kuhn, M.R., Bagi, K.: On the relative motions of two rigid bodies at a compliant contact: application to granular media. Mech. Res. Commun. 32(4), 463-480 (2005)

14. Kuhn, M.R., Chang, C.S.: Stability, bifurcation, and softening in discrete systems: a conceptual approach for granular materials. Int. J. Solids Struct. 43(20), 6026-6051 (2006)

15. Lafond, V., Cordonnier, T., De Coligny, F., Courbaud, B.: Reconstructing harvesting diameter distribution from aggregate data. Ann. For. Sci. 69(2), 235-243 (2012)

16. Laouafa, F., Darve, F.: Modelling of slope failure by a material instability mechanism. Computer. Geotech. 29(4), 301-325 (2002)

17. Luding, S.: Micro-macro transition for anisotropic, frictional granular packings. Int. J. Solids Struct. 41, 5821-5836 (2004)

18. Nicot, F., Darve, F., Koah, H.-D.-V.: Bifurcation and second-order work in geomaterials. Int. J. Num. Anal. Methods Geomech. 31, 1007-1032 (2007)

19. Nicot, F., Hadda, N., Bourrier, F., Sibille, L., Darve, F.: Failure mechanisms in granular media: a discrete element analysis. Granul. Matter 13(3), 255-260 (2011)

20. Nicot, F., Hadda, N., Bourrier, F., Sibille, L., Wan, R., Darve, F.: Inertia effects as a possible missing link between micro and macro second-order work in granular media. Int. J. Solids Struct. 49(10), 1252-1258 (2012a)

21. Nicot, F., Sibille, L., Darve, F.: Bifurcation in granular materials: an attempt at a unified framework. Int. J. Solids Struct. 46, 3938-3947 (2009)

22. Nicot, F., Sibille, L., Darve, F.: Failure in rate-independent granular materials as a bifurcation toward a dynamic regime. Int. J. Plast. 29, 136-154 (2012b)

23. Nicot, F., Darve, F.: Diffuse and localized failure modes: two competing mechanisms. Int. J. Numer. Anal. Meth. Geomech. 35(5), 586-601 (2011) 
24. Oda, M., Iwashita, K.: Study on couple stress and shear band development in granular media based on numerical simulation analyses. Int. J. Eng. Sci. 38(15), 1713-1740 (2000)

25. Oda, M., Junichi, K., Siavouche, N.-N.: Experimental micromechanical evaluation of strength of granular materials effects of particle rolling. Mech. Mater. 1, 269-283 (1982)

26. Oda, M., Takemura, T., Takahashi, M.: Microstructure of shear band developed in Toyoura sand by means of microfocus X-ray computed tomography. Géotechnique 54, 539-542 (2004)

27. Pearson, K.: On the criterion that a given system of deviation from the probable in the case of a correlated system of variables is such that it can be reasonably supposed to have arisen from random sampling. Philos. Mag. Ser. 5(50), 157-175 (1900)

28. Radjai, F., Roux, S., Moreau, J.J.: Contact forces in a granular packing. CHAOS 9(3), 544 (1999)

29. Radjai, F., Wolf, D.E., Jean, M., Moreau, J.J.: Bimodal character of stress transmission in granular packings. Phys. Rev. Lett. 80(1), 61-64 (1998)

30. Sibille, L., Donzé, F.V., Nicot, F., Darve, F.: Analysis of failure occurrence from direct simulations. Eur. J. Environ. Civ. Eng. 13(2), 187-202 (2009)

31. Sibille, L., Donzé, F.-V., Nicot, F., Chareyre, B., Darve, F.: Bifurcation detection and catastrophic failure. Acta Geotecnica 3, 14-24 (2008)

32. Steele, M., Chaseling, J.: Powers of discrete goodness-of-fit test statistics for a uniform null against a selection of alternative distributions. Commun. Stat. Simul. Comput. 35(4), 1067-1075 (2006)
33. Tordesillas, A.: Force chain buckling, unjamming transitions and shear banding in dense granular assemblies. Philos. Mag. A Phys. Condens. Matter Struct. Defects Mech. Prop. 87(32), 4987-5016 (2007)

34. Tordesillas, A., Muthuswamy, M.: On the modeling of confined buckling of force chains. J. Mech. Phys. Solids 57(4), 706-727 (2009)

35. Tordesillas, A., Lin, Q., Zhang, J., Behringer, R.P., Shi, J.: Structural stability and jamming of self-organized cluster conformations in dense granular materials. J. Mech. Phys. Solids 59(2), 265-296 (2011). Elsevier

36. Tordesillas, A., O’Sullivan, P., Walker, D.M.: Evolution of functional connectivity in contact and force chain networks: Feature vectors, $\mathrm{k}$-cores and minimal cycles. Comptes Rendus Mécanique 338(10-11), 556-569 (2010). Elsevier Masson SAS

37. Tordesillas, A., Walker, D.M., Lin, Q.: Force cycles and force chains. Phys. Rev. E 81, 011302 (2010)

38. Walker, D.M., Tordesillas, A.: Topological evolution in dense granular materials: a complex networks perspective. Int. J. Solids Struct. 47(5), 624-639 (2010)

39. Welker, P., McNamara, S.: Precursors to failure and weakening in a biaxial test. Granul. Matter 13, 93-105 (2011)

40. Šmilauer, V., Catalano, E., Chareyre, B., Dorofeenko, S., Duriez, J., Gladky, A., Kozicki, J., et al.: Yade Documentation, The Yade Project. (2010) Retrieved from http://yade-dem.org/doc/ 